\title{
Bridging the Divide Between HPC and Commodity System Software
}

\author{
(Invited Talk) \\ John R. Lange \\ Department of Computer Science \\ University of Pittsburgh \\ jacklange@cs.pitt.edu
}

\begin{abstract}
Cloud computing holds great promise for High Performance Computing (HPC) users and applications. Accordingly a large amount of work has gone into exploring and enabling the use of current cloud service architectures to support HPC applications. While the allure of cloud based HPC systems is very compelling, there are still a number of issues that prevent the cloud from becoming a truly viable HPC platform. In particular, application performance has been found to suffer from competing workloads, randomized layouts and node assignments, as well as competing network flows. All of these issues arise from the fact that HPC applications are forced to share and compete for resources along with a wide variety of other commodity applications. Unfortunately, the presence of these competing workloads is critical to the success of the cloud model, which relies on the economics of leveraging shared resources. While this inherent tension has so far acted as a barrier to the deployment of HPC applications in the cloud, we claim that it can be overcome using multiple specialized system software stacks that are capable of providing isolated partitions for co-located workloads. This talk will focus on the design and development of system software capable of effectively supporting HPC applications in a commodity cloud environment through the use of dynamic resource partitioning and isolated management layers.
\end{abstract}

\section{Categories and Subject Descriptors}

D.4.7 [Operating Systems]: Organization and Design

\section{General Terms}

Design, Performance, Experimentation

\section{Keywords \\ Operating Systems, Virtualization, Exascale}

\section{Speaker Bio}

John Lange is an Assistant Professor of Computer Science and Computer Engineering at the University of Pittsburgh. Before joining the faculty at Pittsburgh he received his M.S. and Ph.D. degrees at Northwestern University where he conducted research in high performance, distributed, and cloud computing. While at Northwestern he developed the Palacios Virtual Machine Monitor, an OS independent virtualization architecture targeting high performance environments. Palacios has been deployed on a large variety of HPC systems including commodity compute clusters and large scale supercomputers. Since moving to Pittsburgh, he has conducted research on specialized high performance system software architectures and approaches for adopting these specialized approaches in commodity cloud environments. During the last few years his work has focused on supporting performance isolation in consolidated environments through the use of multiple independent specialized system software stacks. His research group has also contributed many of the underlying system software components for the next generation exascale OS/R prototype being developed by the Hobbes project for the Department of Energy.

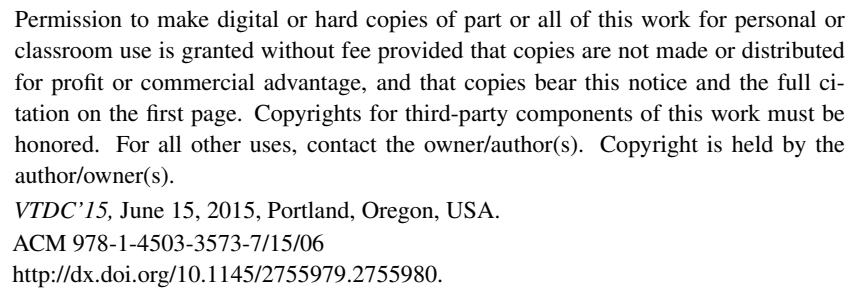

Permission to make digital or hard copies of part or all of this work for personal or classroom use is granted without fee provided that copies are not made or distributed for profit or commercial advantage, and that copies bear this notice and the full citation on the first page. Copyrights for third-party components of this work must be honored. For all other uses, contact the owner/author(s). Copyright is held by the author/owner(s).

VTDC'15, June 15, 2015, Portland, Oregon, USA.

ACM 978-1-4503-3573-7/15/06

http://dx.doi.org/10.1145/2755979.2755980. 